POS PROCEEDINGS

\title{
Bow shock sources close to the Galactic centre
}

\author{
Michal Zajaček* \\ Center for Theoretical Physics, Polish Academy of Sciences, Al. Lotnikow 32/46, 02-668 \\ Warsaw, Poland \\ I. Physikalisches Institut der Universität zu Köln, Zülpicher Strasse 77, D-50937 Köln, Germany \\ Max-Planck-Institut für Radioastronomie (MPIfR), Auf dem Hügel 69, D-53121 Bonn, Germany \\ E-mail: zajacek@ph1.uni-koeln.de
}

\section{Andreas Eckart, Seyedeh Elaheh Hosseini}

I. Physikalisches Institut der Universität zu Köln, Zülpicher Strasse 77, D-50937 Köln, Germany Max-Planck-Institut für Radioastronomie (MPIfR), Auf dem Hügel 69, D-53121 Bonn, Germany

\begin{abstract}
We provide an up-to-date summary of the current observational and theoretical studies of stellar bow-shock sources close to the Galactic centre. The symmetry axis of a bow shock provides the information on the relative motion of the star with respect to the ambient medium, while the photometry and spectroscopy in NIR domain give information about the 3D motion of the star. Hence, it is possible from this data to obtain an estimate on the motion of the ambient medium. In combination with the estimate of the bow-shock size, it is possible to infer the valuable information on the density of the hot accretion flow close to the Galactic centre. In particular, we outline a statistical method to determine the ambient density slope based on either multiple bowshock detections for one star along its orbit or multiple bow-shock detections for several sources at different distances from Sgr A*.
\end{abstract}

Accretion Processes in Cosmic Sources - II - APCS2018

3-8 September 2018

Saint Petersburg, Russian Federation

${ }^{*}$ Speaker. 


\section{Introduction}

The Galactic centre environment represents a unique astrophysical laboratory where one can study the dynamical effects inside one of the densest stellar clusters on one hand (Alexander 2005; Merritt 2013) and it also allows us to study the mutual interaction between stars and the gaseousdusty medium inside the sphere of influence of compact radio source Sgr A* on the other hand (see Genzel et al. 2010; Zajaček et al. 2017b; Eckart et al. 2017, for overviews).

The Galactic centre is characterized by an overall increase in the NIR and MIR surface brightness along the Galactic plane (Becklin \& Neugebauer 1968, 1969; Low et al. 1969). The NIR emission is dominated by the photosphere emission of individual stellar sources that constitute a crowded stellar field, while the MIR emission originates in both the diffuse gaseous-dusty circumnuclear medium and the dusty circumstellar envelopes of a few stellar sources.

In terms of stellar populations, there are both late-type stars whose number density forms a flat core towards the centre and early-type stars that form a steeper cusp (Buchholz et al. 2009). There are clear signatures that recent in situ star-formation event took place inside the sphere of influence of the supermassive black hole (SMBH) associated with Sgr A*. This is mainly based on the three populations of massive young OB stars in the innermost parsec (Sanchez-Bermudez et al. 2014):

- scattered population at the distance of 0.04 up to 1.2 parsecs (hereafter pc),

- disc-like population at $0.04 \mathrm{pc}$ up to $0.5 \mathrm{pc}$,

- a population of fast-moving, lighter B stars inside $0.04 \mathrm{pc}$, which is also known as the $\mathrm{S}$ cluster (Eckart \& Genzel 1996, 1997).

These stars have large mass-loss rates of as much as $\dot{M}_{\mathrm{w}} \approx 10^{-7} M_{\odot} \mathrm{yr}^{-1}-10^{-5} M_{\odot} \mathrm{yr}^{-1}$ and highvelocity stellar winds $v_{\mathrm{w}} \approx 10^{3} \mathrm{~km} \mathrm{~s}^{-1}$ and they can, therefore, strongly interact with the surrounding medium.

Inside the sphere of influence, $r_{\text {inf }} \sim 1.7 \mathrm{pc}$ for $\mathrm{Sgr} \mathrm{A}^{*}$, stars orbit the SMBH approximately with Keplerian velocities,

$$
v_{\star} \simeq 415\left(\frac{M_{\bullet}}{4 \times 10^{6} M_{\odot}}\right)^{1 / 2}\left(\frac{r}{0.1 \mathrm{pc}}\right)^{-1 / 2} \mathrm{~km} \mathrm{~s}^{-1} \text { for } r \lesssim r_{\text {inf }},
$$

where $r$ is the distance from Sgr $\mathrm{A}^{*}$. The basic condition for the bow-shock formation in the circumnuclear gaseous-dusty medium is that the relative velocity of the star with respect to the ambient medium $\mathbf{v}_{\text {rel }}=\mathbf{v}_{\star}-\mathbf{v}_{\mathrm{a}}$ is larger than the local speed at which signals and perturbations propagate in the medium. For the non-magnetized or weakly magnetized medium, the wave speed is the local sound speed $c_{\mathrm{s}}(r)$,

$$
c_{\mathrm{s}}=482\left(\frac{\gamma}{1.4}\right)^{1 / 2}\left(\frac{T_{\mathrm{a}}}{10^{7} \mathrm{~K}}\right)^{1 / 2}\left(\frac{\mu_{\mathrm{HII}}}{0.5}\right)^{-1 / 2} \mathrm{~km} \mathrm{~s}^{-1},
$$

where we have assumed that the ambient medium is fully ionized. The temperature of the ambient medium $T_{\mathrm{a}}$ is based on the X-ray observations on the scale of the Bondi radius (Baganoff et al. 2003; Wang et al. 2013). By dividing Equations 1.1 and 1.2, we essentially obtain the Mach speed $\mathscr{M} \equiv v_{\text {rel }} / c_{\text {s }}$, which is close to unity at the Bondi radius. An additional inflow or outflow, especially 
approximately perpendicular to the orbital velocity, can increase the relative velocity, which sets the Mach speed above unity and the bow-shock formation can occur in the ambient non-magnetized medium. It should be noted that even for the subsonic relative velocities, the supersonic stellar wind gets shocked and due to a non-zero relative speed, an elongated closed cavity forms separated by the shocked stellar-wind layer from the surrounding interstellar medium.

There are several indications that the Galactic centre environment is strongly magnetized at both larger and smaller scales (see in particular Yusef-Zadeh \& Morris 1987; Eatough et al. 2013; Gravity Collaboration 2018). Multiwavelength measurements of the Galactic centre magnetar involving the Faraday rotation measurement (Eatough et al. 2013) revealed a strong magnetic field of $B \geq 50 \mu \mathrm{G}$ at the projected distance scale of $\sim 3^{\prime \prime}=0.12 \mathrm{pc}$. For the Galactic centre plasma, such a strong field is dynamically crucial and implies that the plasma at the Bondi radius is magnetically dominated. In combination with the number density of $n_{\mathrm{e}}^{B}=18.3 \pm 0.1 \mathrm{~cm}^{-3}$ at the Bondi radius (Różańska et al. 2015), it is possible to estimate the Alfvén velocity at which disturbances propagate,

$$
v_{\mathrm{A}}=\sqrt{\frac{B^{2}}{\mu_{0} \mu m_{\mathrm{H}} n_{\mathrm{e}}^{\text {out }}}} \approx 36\left(\frac{B}{50 \mu G}\right)\left(\frac{\mu_{\mathrm{HII}}}{0.5}\right)^{-1 / 2}\left(\frac{n_{\mathrm{e}}^{\text {out }}}{18.3 \mathrm{~cm}^{-3}}\right)^{-1 / 2} \mathrm{~km} \mathrm{~s}^{-1} .
$$

Dividing Eq. (1.1) by Eq. 1.3 defines the Alfvénic Mach speed, $\mathscr{M}_{\mathrm{A}} \equiv v_{\text {rel }} / v_{\mathrm{A}}$, which turns out to be larger than unity in the magnetically dominated plasma. For a more detailed investigation, see also Zajaček et al. (2017b) and Fig. 1 therein.

Based on the theoretical estimates of the Mach speed, the formation of stellar bow shocks is expected in the innermost parsec of the Galactic centre. Several of them have been detected as comet-shaped structures in the NIR-, but more prominently in MIR-domains (Tanner et al. 2002; Rigaut et al. 2003; Geballe et al. 2004; Tanner et al. 2005; Mužić et al. 2010; Buchholz et al. 2011, 2013; Rauch et al. 2013; Sanchez-Bermudez et al. 2014), where the emission of dust of $\sim 1000 \mathrm{~K}$ becomes more prominent. Recently, we also showed how it is possible to constrain the bow-shock properties for fainter, unresolved NIR-excess sources in the inner S cluster (Eckart et al. 2013), specifically for the fast-moving DSO/G2 source. This can be done by using the polarized signal in the NIR-domain in combination with the 3D Monte Carlo radiative transfer modelling (Shahzamanian et al. 2016; Zajaček et al. 2017a). The orientation of a bow shock and its characteristics (surface density, velocity field) also strongly depend on the presence and the magnitude of an inflow/outflow (Zajaček et al. 2016). In particular, the presence of an ambient outflow of the order of $\sim 1000 \mathrm{~km} \mathrm{~s}^{-1}$ from the direction of Sgr A*, which is inferred from the orientation and the size of comet-shaped sources X3 and X7 (Mužić et al. 2010), induces an asymmetry in the evolution of the stellar bow-shock size and its other characteristics along the orbit of a stellar source.

In this contribution, we analyze the potential of bow shocks in constraining the properties of the hot accretion flow around Sgr A*. The article is structured as follows: in Section 2 we provide an overview of already detected and studied bow-shock sources, subsequently in Section 3 we summarize the basic aspects of constraining the accretion flow with stars, with the focus on using $\mathrm{X}$-ray and radio flares (sub-Section 3.1) and on using bow-shock sizes at different locations along the stellar orbit to infer the density slope (sub-Section 3.2). This is extended in Section 4 for more bow-shock detections along the orbit of one star and for multiple bow-shock detections for several different sources. We provide a short summary in Section 5. 


\section{Overview of detected bow shock sources}

In Table 1, we list previously detected NIR- and MIR-sources that have been resolved and show an elongated, bow-shock morphology. In general, apart from the elongated morphology for detected sources, bow-shock sources exhibit a NIR-excess from NIR towards MIR wavelengths, which can be simply interpreted by the reprocessing of UV emission of a star by a surrounding dust (Viehmann et al. 2006). Bow shocks also exhibit a prominent linear polarization, whose degree rises from NIR to MIR wavelengths, which is caused by dust scattering, overall non-spherical geometry and a likely alignment of dust grains by magnetic field (Buchholz et al. 2013), which is a prominent feature in terms of linear polarization along the minispiral arms (Roche et al. 2018).

\begin{tabular}{c|c|c|c}
\hline \hline Name & Coordinates & Projected offset (Sgr A*) & Publication \\
\hline X3 & $17^{\mathrm{h}} 45^{\mathrm{m}} 39.85^{\mathrm{s}},-29^{\circ} 00^{\prime} 30.5^{\prime \prime}$ & $3.4^{\prime \prime}$ & {$[1]$} \\
X7 & $17^{\mathrm{h}} 45^{\mathrm{m}} 40.00^{\mathrm{s}},-29^{\circ} 00^{\prime} 28.6^{\prime \prime}$ & $0.8^{\prime \prime}$ & {$[1]$} \\
IRS 1W & $17^{\mathrm{h}} 45^{\mathrm{m}} 40.442^{\mathrm{s}},-29^{\circ} 00^{\prime} 27.51^{\prime \prime}$ & $5.3^{\prime \prime}$ & {$[2]$} \\
IRS 5 & $17^{\mathrm{h}} 45^{\mathrm{m}} 40.700^{\mathrm{s}},-29^{\circ} 00^{\prime} 18.51^{\prime \prime}$ & $13.1^{\prime \prime}$ & {$[2]$} \\
IRS 10W & $17^{\mathrm{h}} 45^{\mathrm{m}} 40.5305^{\mathrm{s}},-29^{\circ} 0023.001^{\prime \prime}$ & $8.3^{\prime \prime}$ & {$[2]$} \\
IRS 21 & $17^{\mathrm{h}} 45^{\mathrm{m}} 40.222^{\mathrm{s}},-29^{\circ} 00^{\prime} 30.85^{\prime \prime}$ & $3.6^{\prime \prime}$ & {$[2]$} \\
IRS 2L & $17^{\mathrm{h}} 45^{\mathrm{m}} 39.778^{\mathrm{s}},-29^{\circ} 00^{\prime} 32.11^{\prime \prime}$ & $5.2^{\prime \prime}$ & {$[3]$} \\
IRS 8 & $17^{\mathrm{h}} 45^{\mathrm{m}} 40.14^{\mathrm{s}},-28^{\circ} 59^{\prime} 58.7^{\prime \prime}$ & $29.1^{\prime \prime}$ & {$[4],[5]$} \\
X24 (MP-09-14.4) & $17^{\mathrm{h}} 45^{\mathrm{m}} 39.424^{\mathrm{s}},-29^{\circ} 00^{\prime} 42.85^{\prime \prime}$ & $17^{\prime \prime}$ & {$[6],[7]$} \\
IRS7 & $17^{\mathrm{h}} 45^{\mathrm{m}} 39.987^{\mathrm{s}},-29^{\circ} 00^{\prime} 22.24^{\prime \prime}$ & $6^{\prime \prime}-7^{\prime \prime}$ & {$[8]$} \\
\hline
\end{tabular}

Table 1: Summary of prominent bow-shocks sources in the inner parsec of the Galactic centre - columns summarize the designation, coordinates, projected offset from Sgr A* ( $1^{\prime \prime}$ corresponds to $\sim 0.04 \mathrm{pc}$ at the Galactic centre), and the publication where the most recent data can be found: [1] Mužić et al. (2007), [2] Sanchez-Bermudez et al. (2014), [3] Viehmann et al. (2006), [4] Geballe et al. (2004), [5] Rauch et al. (2013), [6] Sabha et al. (2014), [7] Zhao et al. (2009), [8] Yusef-Zadeh \& Morris (1991).

The importance of stellar bow shocks in the Galactic center lies in the fact that they can be employed as

(a) probes of circumnuclear ambient density and interstellar medium velocity in case the stellar parameters are known, which directly follows from the stand-off distance relation (Wilkin 1996),

$$
R_{0} \sim\left(\frac{\dot{m}_{\mathrm{w}} v_{\mathrm{w}}}{4 \pi \rho_{\mathrm{a}} v_{\mathrm{rel}}^{2}}\right)^{1 / 2}
$$

where $\rho_{\mathrm{a}}$ is the ambient density, $v_{\text {rel }}$ is the relative velocity of the star with respect to the ambient medium, $\dot{m}_{\mathrm{w}}$ is the mass-loss rate of the star and $v_{\mathrm{w}}$ is the terminal velocity of its stellar wind,

(b) on the other hand, they can be used for estimating stellar properties in case the ambient medium density and its velocity are known alongside the stellar velocity, which again follows from Eq. (2.1), 
(c) combining approaches outlined in points (a) and (b), they can provide extra information on the dynamics of the nuclear star cluster. In particular, Sanchez-Bermudez et al. (2014) found that the bow-shock sources IRS 21, IRS 1W, IRS 5, and IRS 10W belong to a scattered population of young massive Wolf-Rayet stars, which are not a part of any coherent stellar structure previously known in the nuclear star cluster, in particular the clockwise stellar disc inside $0.5 \mathrm{pc}$,

(d) they can be used as probes of the hot accretion flow inside the Bondi radius of Sgr A*, i.e. within the $\mathrm{S}$ cluster, as we will discuss in more detail below.

\section{Probing accretion flow around Sgr A* with stars}

Compact radio source $\mathrm{Sgr} \mathrm{A}^{*}$ is surrounded by one of the densest as well as the oldest stellar clusters in the Galaxy (Schödel et al. 2014). The stellar motion is dominated by the potential of Sgr A* inside the sphere of the gravitational influence of the black hole, $r_{\mathrm{h}} \equiv G M_{\bullet} / \sigma_{\star}^{2}$, where $\sigma_{\star}$ is the one-dimensional stellar velocity dispersion, which can be distance-dependent. Therefore, kinematically it is easier to describe the SMBH potential dominance by the radius beyond which the mass of the central black hole is smaller than the enclosed stellar mass, $M_{\bullet} \lesssim M_{\mathrm{enc}}\left(r>r_{\mathrm{h}}\right)$, which is about 2 pc for the Galactic centre Nuclear Stellar Cluster (Merritt 2013). Inside the influence radius, one can treat the stellar velocities as Keplerian as the first approximation, see Eq. (1.1).

The hot ambient gas can be captured by Sgr A* at smaller scales of $1^{\prime \prime}-2^{\prime \prime}$, which is given by the Bondi radius for steady, spherical accretion,

$$
R_{\mathrm{B}}=\frac{G M \bullet}{c_{\mathrm{S}}^{2}} .
$$

The accretion flow inside the Bondi radius and its associated radiative properties are generally given by radiatively inefficient accretion flow solutions (RIAFs, ). The RIAF region inside Bondi radius coincides with the innermost cluster of fast B-type stars - so-called S cluster (Eckart \& Genzel 1996, 1997) - that is mostly located inside the innermost arcsecond, which corresponds to $\sim 0.04 \mathrm{pc}$ for the Galactic centre. This provides an opportunity to test the accretion flow with stars by studying,

- their potential bow-shocks as they interact with the surrounding medium, which could be revealed in the infrared, the X-ray or the radio domain, as was modelled by several authors (Christie et al. 2016; Zajaček et al. 2016, 2017a; Ballone et al. 2018; Schartmann et al. 2018),

- longer X-ray flares which could result from stellar photons Compton-upscattered in the inner portions of the hot accretion flow (Nayakshin 2005).

In case the stellar bow shocks are resolved for the inner S stars, their properties are expected to vary quite significantly along their orbit. For instance, the stand-off distance given by Eq. (2.1) assuming the equilibrium between the ram and wind pressure, varies between the apocentre and the pericentre of the orbit as,

$$
\frac{R_{0 A}}{R_{0 P}}=\left(\frac{r_{\mathrm{p}}}{r_{\mathrm{a}}}\right)^{-\gamma / 2}\left(\frac{v_{\star \mathrm{p}}}{v_{\star \mathrm{a}}}\right)
$$


where $\gamma$ is the power-law slope of the accretion flow, whose number density is simply given as $n_{\mathrm{a}}=$ $n_{0}\left(r / r_{0}\right)^{-\gamma}$ with $\gamma>0$, and in the further derivations, we assume that the stellar relative velocity can be approximated with the stellar velocity as given by the vis-viva integral, $v_{\star}=\sqrt{G M_{\bullet}(2 / r-1 / a)}$. Then the ratio in Eq. (3.2) can be expressed elegantly only as the function of the orbital eccentricity and the density slope,

$$
\frac{R_{0 A}}{R_{0 P}}=\left(\frac{1+e}{1-e}\right)^{\gamma / 2+1}
$$

Similarly, the ratio between the latus-rectum orbital position (the true anomaly of $90^{\circ}$ ) and the pericentre can simply be derived as,

$$
\frac{R_{0 L}}{R_{0 P}}=\frac{(1+e)^{\gamma / 2+1}}{\left(1+e^{2}\right)^{1 / 2}} .
$$

Hence, it follows from Eqs. (3.3) and (3.4) and for non-zero eccentricity, there is always a bow-shock size variation, even for a constant ambient density with $\gamma=0$. For instance, for the short-period S2 star whose eccentricity is currently well-constrained to be $e=0.884$, we get the apocentre-pericentre variation of $R_{0 A} / R_{O P} \approx 65$ and the latus-rectum-pericenre variation of $R_{0 L} / R_{0 P} \approx 2$ for the inferred RIAF density slope close to $\gamma \approx 1$ (Wang et al. 2013), which is also illustrated in Fig. 1 for the analytical thin-shell bow-shock solution (see Wilkin 1996). Since the radiative properties of bow shocks are proportional to their linear size, this motivates to search for potential flux density changes for high-eccentricity $S$ stars, for which the changes along their orbit are most pronounced. According to Fig. 1, the bow-shock is the smallest at the pericentre of a stellar orbit due to the largest ram-pressure, but at the same time it will have the largest luminosity.

\subsection{Inferring density from the (non)detection of radio and X-ray flares}

It was suggested using both analytical and numerical approaches that the interaction of the stellar wind of the fast-moving S2 star with the accretion flow should lead to the detectable monthlong X-ray flare with the peak luminosity of $L_{\mathrm{X}} \approx 4 \times 10^{33} \mathrm{erg} \mathrm{s}^{-1}$ due to the increased thermal X-ray bremsstrahlung originating in the bow-shock (Giannios \& Sironi 2013; Christie et al. 2016). Such an increase is comparable to the quiescent X-ray emission of the Sgr A* accretion flow and could be marginally detected with the current X-ray instruments. In principle, it could help constrain the accretion flow properties (density, density slope, its thickness) at the distance of the order of 1000 Schwarzschild radii $\left(r_{\mathrm{s}}\right)$, which is in general an unprobed, intermediate region (Gillessen et al. 2019) between the Bondi radius region of $10^{5} r_{\mathrm{s}}$ probed in soft X-ray thermal emission (Baganoff et al. 2003) and the region on the scale of $10-100 r_{\mathrm{s}}$ probed in the submmdomain using the polarized emission (Bower et al. 2003; Marrone et al. 2007). Another contribution to the X-ray flux comes from the UV/optical photons of the S2 star that are Compton-upscattered in the inner hot RIAF region (Nayakshin 2005). Based on the non-detection of any month-long X-ray flare during S2 pericenter-passages in 2002 and 2018, the density structure and the slope of the accretion flow are fully consistent with the hot, diluted RIAF solution of Yuan et al. (2003) with the upper limit on the accretion rate of $\dot{M}_{\text {acc }} \approx 2 \times 10^{-7} M_{\odot} \mathrm{yr}^{-1}$ (Marrone et al. 2007). This is also consistent with the updated 3D AMR hydrodynamical simulations of the interaction between the 


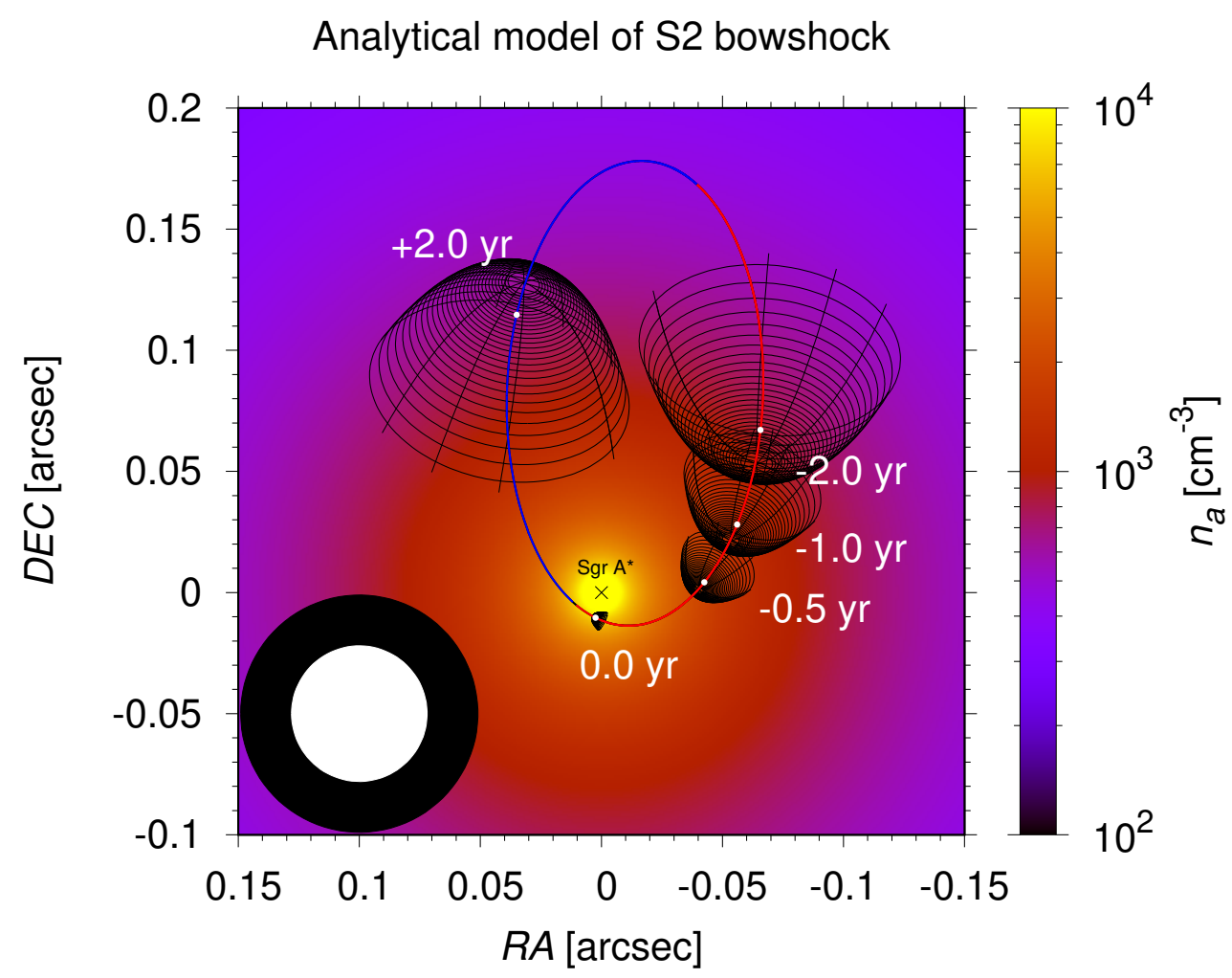

Figure 1: Bow-shock size evolution of S2 star orbiting Sgr A* calculated according to the thin-shell analytical solution by Wilkin (1996). Colour-coded background expresses RIAF density profile according to the solution by Yuan et al. (2003) and Xu et al. (2006): $n_{\mathrm{a}} \approx 10^{4}\left(\mathrm{r} / 10^{15} \mathrm{~cm}\right)^{-1} \mathrm{~cm}^{-3}$. Two concentric black and white circles in the lower left corner express the diffraction-limit resolution with eight-meter class telescopes in the NIR $L^{\prime}$ and $K_{\mathrm{s}}$-band, respectively. For S2 star, the bow-shock would be unresolved close to the pericentre of its orbit, but it could be revealed via the spectral changes of Sgr A*-S2 system during the pericentre passage.

S2 stellar wind and the accretion flow, which predicted no significant X-ray emission excess close to the S2 pericentre passage (Schartmann et al. 2018).

The detected dusty source G2 or DSO (Gillessen et al. 2012; Eckart et al. 2013) was also predicted to drive a shock into the ambient medium, which in principle could lead to the detectable nonthermal radio synchrotron emission (Narayan et al. 2012). This theory can be expanded in a straightforward way to all S stars, including S2 (Ginsburg et al. 2016). The general prediction was that the electrons in the accretion flow are accelerated in the bow-shock region to relativistic energies, with the peak flux close to $1 \mathrm{GHz}$. Concerning stellar bow shocks, the main sources of uncertainty are the bow-shock size and the magnetic field strength that is enhanced in the shocked region. However, for realistic values of the parameters applied to DSO/G2 case, the radio synchrotron emission was found to be well below the quiescent radio emission of Sgr A* (Crumley \& Kumar 2013; Zajaček et al. 2016). For S2 star, Ginsburg et al. (2016) show that its synchrotron flux would be comparable to that of $\mathrm{Sgr} \mathrm{A}^{*}$ at $10 \mathrm{GHz}$ (radio band) as well as $10^{14} \mathrm{~Hz}$ (infrared) for extreme combinations of the mass-loss rate and the wind velocity: $\left(\dot{m}_{\mathrm{w}}, v_{\mathrm{w}}\right)=\left(10^{-5} \mathrm{M}_{\odot} \mathrm{yr}^{-1}, 1000 \mathrm{~km} \mathrm{~s}^{-1}\right)$ 
and $\left(\dot{m}_{\mathrm{w}}, v_{\mathrm{w}}\right)=\left(10^{-6} M_{\odot} \mathrm{yr}^{-1}, 4000 \mathrm{~km} \mathrm{~s}^{-1}\right)$.

\subsection{Density slope determination for one bow-shock source orbiting Sgr A*}

The bow-shock length-scale is given by the stagnation (stand-off) distance $R_{0}$, which can be derived under the assumption that in the stationary case, the stellar wind pressure is equal to the sum of the ram pressure and the thermal pressure, $P_{\mathrm{w}}=P_{\mathrm{ram}}+P_{\mathrm{th}}=P_{\mathrm{ram}}(1+\alpha)$, where the last equality is obtained using the ratio $\alpha=P_{\text {th }} / P_{\text {ram }}$. In the most general case, the stagnation distance of the bow-shock, i.e. the distance from the star to the tip of the bow shock, can be expressed as (Wilkin 1996; Zhang \& Zheng 1997; Christie et al. 2016),

$$
R_{0}=\left(\frac{\dot{m}_{\mathrm{w}} v_{\mathrm{w}}}{\Omega_{\mathrm{w}} \rho_{\mathrm{a}} v_{\text {rel }}^{2}(1+\alpha)}\right)^{1 / 2} \simeq C_{\star} \rho_{\mathrm{a}}^{-1 / 2} v_{\text {rel }}^{-1},
$$

where the last equality is obtained under the assumption that the thermal pressure is negligible, i.e. $\alpha \rightarrow 0$, which is essentially met for the case of highly-supersonic motion, $\mathscr{M} \equiv v_{\text {rel }} / c_{\mathrm{s}}=$ $1 / \sqrt{v \alpha} \gg 1$ for $\alpha \rightarrow 0$ ( $v$ is the adiabatic index). The quantity $C_{\star}^{2}=\dot{m}_{\mathrm{w}} v_{\mathrm{w}} / \Omega_{\mathrm{w}}$ represents the momentum flux of the stellar wind per unit solid angle $\Omega_{\mathrm{w}}$, which is equal to $\Omega_{\mathrm{w}}=4 \pi$ for an isotropic outflow. The parameter $C_{\star}$ may be assumed to be constant for several stellar orbits. We estimate the typical value for the brightest star in the S cluster - S2 based on its stellar parameters (Martins et al. 2008),

$$
C_{\star}^{2}=7.85 \times 10^{-5}\left(\frac{\dot{m}_{\mathrm{w}}}{10^{-7} M_{\odot} \mathrm{yr}^{-1}}\right)\left(\frac{v_{\mathrm{w}}}{1000 \mathrm{~km} \mathrm{~s}^{-1}}\right) M_{\odot} \mathrm{km} \mathrm{s}^{-1} \mathrm{yr}^{-1} \mathrm{sr}^{-1}
$$

In the further analysis, we neglect the thermal pressure as well as the motion of the ambient medium. Hence, the relative velocity can be approximated by stellar orbital velocity, $v_{\text {rel }} \simeq v_{\star}$. The ratio of the bow-shock stand-off distances, $R_{01}$ and $R_{02}$, for two corresponding distances of the star from $\mathrm{Sgr} \mathrm{A}^{*}, r_{1}$ and $r_{2}$ (where $r_{1}>r_{2}$ ), can then be expressed as,

$$
\frac{R_{01}\left(r_{1}\right)}{R_{02}\left(r_{2}\right)}=\left(\frac{n_{\mathrm{a} 2}}{n_{\mathrm{a} 1}}\right)^{1 / 2}\left(\frac{v_{\mathrm{rel} 2}}{v_{\mathrm{rel} 1}}\right)=\left(\frac{r_{1}}{r_{2}}\right)^{\gamma / 2}\left(\frac{v_{\star 2}}{v_{\star 1}}\right),
$$

where we adopted the power-law density profile for the ambient medium, $\rho_{\mathrm{a}}=\mu m_{\mathrm{H}} n_{0}\left(r / r_{0}\right)^{-\gamma}$, where $\gamma>0$ is the power-law slope.

The ratio expressed by Eq. (3.7) can be further expanded using the general relation for the Keplerian orbital velocity along the elliptical orbit, $v_{\star}=\sqrt{G M_{\bullet}(2 / r-1 / a)}$, where $r$ is the radial distance from $\mathrm{Sgr} \mathrm{A}^{*}$ and $a$ is a semi-major axis of the elliptical orbit. We get,

$$
\frac{R_{01}}{R_{02}}\left(r_{1}, r_{2}, a, \gamma\right)=\left(\frac{r_{1}}{r_{2}}\right)^{(\gamma+1) / 2}\left(\frac{2 a-r_{2}}{2 a-r_{1}}\right)^{1 / 2} .
$$

It is then trivial to express the power-law slope from Eq. (3.8). We denote it as $\gamma_{12}$ as it is essentially based on bow-shock sizes at two points,

$$
\gamma_{12}=\frac{2 \log \left(R_{01} / R_{02}\right)+\log \left[\left(2 a-r_{1}\right) /\left(2 a-r_{2}\right)\right]}{\log \left(r_{1} / r_{2}\right)}-1 .
$$




\section{Statistical approach}

Now we will present an analytical method for determining the density power-law slope of the ambient medium, in our case hot gas close to the Galactic centre, for multiple bow-shock detections. There are two plausible observable cases:

(i) multiple bow-shock detections for one star (multiple epochs along one orbit),

(ii) multiple bow shock detections for different stars (single epoch sufficient).

In Fig 2 we illustrate both cases - (i) one star (left panel) and (ii) more bow shocks associated with different stars (right panel).
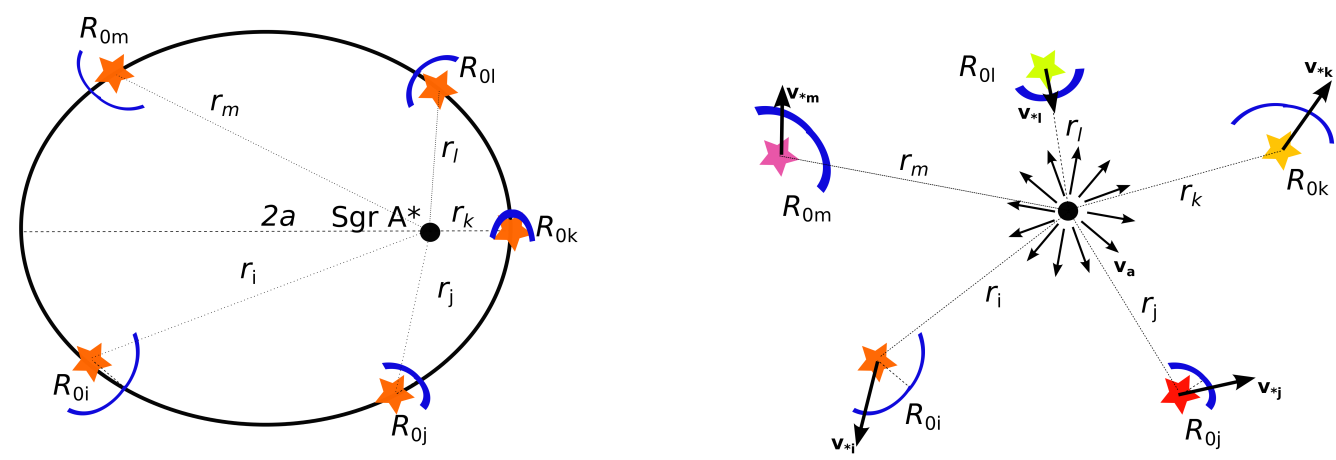

Figure 2: Illustration of a statistical approach for constraining the power-law slope of a spherical density distribution close to Sgr A*: Left panel: Multiple bow shock detections of one star along an elliptical orbit. Right panel: Multiple bow shock detections for different stars with different mass-loss rates $\dot{m}_{\mathrm{w} i}$ and terminal stellar wind velocities $v_{\mathrm{w} i}$. In this case also deprojected stellar orbital velocities $\mathbf{v}_{\star \mathbf{i}}$ and the outflow vector field $\mathbf{v}_{\mathrm{a}}$ are labelled for illustration.

This will make the analysis more practical in terms of actual observations. In the following analysis, we will also include a potential non-negligible motion of the ambient medium described in general by a vector field $\mathbf{v}_{\mathrm{a}}(r, \boldsymbol{\theta}, \phi)$. Hence, the relative velocity of the star with respect to the medium is $\mathbf{v}_{\text {rel }}=\mathbf{v}_{\star}-\mathbf{v}_{\mathrm{a}}$ and the magnitude is $v_{\text {rel }}=\sqrt{\mathbf{v}_{\text {rel }} \cdot \mathbf{v}_{\text {rel }}}=\sqrt{v_{\star}^{2}+v_{\mathrm{a}}^{2}-2 v_{\star} v_{\mathrm{a}} \cos \zeta}$, where $\zeta$ is the angle between the velocity vector of the star and the medium at a given point.

\subsection{Multiple bow-shock detections for one star}

Let us suppose that the bow shock was detected and its deprojected standoff distance $R_{0 i}$ inferred for $N$ locations along the orbit. Then we can calculate the density slope $\gamma_{i j}$ for any two locations $i$ and $j$, where $\{i, j\} \in\{1, \ldots, N\}$, according to $\mathrm{Eq}$ (3.9),

$$
\gamma_{i j}=\frac{2 \log \left[R_{0 i}\left(r_{i}\right) / R_{0 j}\left(r_{j}\right)\right]+\log \left[\left(2 a-r_{i}\right) /\left(2 a-r_{j}\right)\right]}{\log \left(r_{i} / r_{j}\right)}-1 .
$$

When the ambient medium has a known non-negligible velocity field $\mathbf{v}_{\mathrm{a}}$ with respect to the star, the formula (4.1) needs to be modified into a more general form, 


$$
\gamma_{i j}=\frac{2 \log \left[R_{0 i}\left(r_{i}\right) / R_{0 j}\left(r_{j}\right)\right]+\log \left[\frac{v_{\star x}^{2}+v_{a i}^{2}-2 v_{\star i} v_{a i} \cos \zeta_{i}}{v_{\star j}^{2}+v_{a j}^{2}-2 v_{\star j} v_{a j} \cos \zeta_{j}}\right]}{\log \left(r_{i} / r_{j}\right)}-1,
$$

where $v_{\star i}, v_{\mathrm{a} i}$, and $\zeta_{i}$ correspond to the stellar orbital velocity, ambient velocity, and the angle between the two velocity vectors, respectively, at position $i$.

For $N$ bow-shock measurements, $N \geq 2$, we have $N(N-1) / 2$ indices $\gamma_{i j}$, from which we can calculate the mean value,

$$
\bar{\gamma}=\frac{2}{N(N-1)} \sum_{\substack{i, j=1 \\ j \neq i}}^{N} \gamma_{i j}
$$

\subsection{Multiple bow-shock detections for different stars}

Since due to large stellar densities in the innermost arcsecond it is difficult to detect a bow shock, a currently more accessible method is to use more distant bow-shock sources at different locations at a single epoch. However, a disadvantage is that for $N$ bow shock sources with approximately isotropic stellar winds, we have $2 N$ more parameters $\left(\dot{m}_{\mathrm{w}}, v_{\mathrm{w}}\right)$ than for a single source.

Having $i=1, \ldots, N$ detected bow shocks at deprojected distances $r_{i}$ from the Galactic centre, for the ratio of standoff radii $R_{\star i}$ and $R_{\star j}$ for any two sources the following relation holds,

$$
\frac{R_{\star i}}{R_{\star j}}=\frac{C_{\star i}}{C_{\star j}}\left(\frac{n_{\mathrm{a}}\left(r_{j}\right)}{n_{\mathrm{a}}\left(r_{i}\right)}\right)^{1 / 2}\left(\frac{v_{\mathrm{rel} j}}{v_{\text {rel } i}}\right) .
$$

As in previous cases, the power-law index $\gamma_{i j}$ may be determined as follows,

$$
\gamma_{i j}=\frac{2\left[\log \left(R_{\star i} / R_{\star j}\right)-\log \left(C_{\star i} / C_{\star j}\right)\right]+\log \left[\frac{v_{\star i}^{2}+v_{a i}^{2}-2 v_{\star i} v_{a i} \cos \zeta_{i}}{v_{\star j}^{2}+v_{a j}^{2}-2 v_{\star j} v_{a j} \cos \zeta_{j}}\right]}{\log \left(r_{i} / r_{j}\right)} .
$$

In case the ambient velocity field is negligible in comparison with stellar velocities, $\mathbf{v}_{\mathrm{a}}=$ $0 \mathrm{~km} \mathrm{~s}^{-1}$, and the orbital velocities at $r_{i}$ are approximately given by Keplerian circular velocities, $v_{i}=\left(G M_{\bullet} / r_{i}\right)^{1 / 2}$, relation (4.5) may be simplified into the form,

$$
\gamma_{i j}=\frac{2\left[\log \left(R_{\star i} / R_{\star j}\right)-\log \left(C_{\star i} / C_{\star j}\right)\right]}{\log \left(r_{i} / r_{j}\right)}-1 .
$$

The mean value of the density power-law profile $\bar{\gamma}$ for $N$ different bow shocks is determined by the relation that is identical to Eq. (4.3).

\section{Summary}

In this contribution, we presented an overview of observational studies of bow-shock sources in the Galactic centre region. About ten stellar bow shocks have been detected and analyzed in the NIR- and MIR-domain where they are the most prominent because of thermal dust emission and scattering. In addition, we outlined that their signatures should also be detected in the X-ray domain due to thermal bremsstrahlung as well as in the radio domain due to non-thermal synchrotron 
emission. However, because of diluted ambient medium in the central parsec of our Galaxy, there have been no successful detections in these domains.

Furthermore, we introduced a simple statistical method to determine the ambient density gradient in the inner stellar cluster region based on either multiple bow-shock detections for one star bound to Sgr A* or bow-shock detections for more stars at different distances from Sgr A*. Although the method is still observationally challenging, since several parameters need to be obtained (at least stand-off radii of bow shocks at different positions and corresponding deprojected radii), stellar bow shocks may become complementary probes of the hot accretion flow around Sgr A* in the era of high-angular resolution when 30-meter telescopes in the NIR- and MIR-domain are operational.

\section{Acknowledgements}

Michal Zajaček acknowledges the financial support from the National Science Centre, Poland, grant No. 2017/26/A/ST9/00756 (Maestro 9).

\section{References}

Alexander, T. 2005, Physics Reports, 419, 65

Baganoff, F. K., Maeda, Y., Morris, M., et al. 2003, ApJ, 591, 891

Ballone, A., Schartmann, M., Burkert, A., et al. 2018, MNRAS, 479, 5288

Becklin, E. E. \& Neugebauer, G. 1968, ApJ, 151, 145

Becklin, E. E. \& Neugebauer, G. 1969, ApJL, 157, L31

Bower, G. C., Wright, M. C. H., Falcke, H., \& Backer, D. C. 2003, ApJ, 588, 331

Buchholz, R. M., Schödel, R., \& Eckart, A. 2009, A\&A, 499, 483

Buchholz, R. M., Witzel, G., Schödel, R., \& Eckart, A. 2013, A\&A, 557, A82

Buchholz, R. M., Witzel, G., Schödel, R., et al. 2011, A\&A, 534, A117

Christie, I. M., Petropoulou, M., Mimica, P., \& Giannios, D. 2016, MNRAS, 459, 2420

Crumley, P. \& Kumar, P. 2013, MNRAS, 436, 1955

Eatough, R. P., Falcke, H., Karuppusamy, R., et al. 2013, Nature, 501, 391

Eckart, A. \& Genzel, R. 1996, Nature, 383, 415

Eckart, A. \& Genzel, R. 1997, MNRAS, 284, 576

Eckart, A., Hüttemann, A., Kiefer, C., et al. 2017, Foundations of Physics, 47, 553

Eckart, A., Mužić, K., Yazici, S., et al. 2013, A\&A, 551, A18 
Geballe, T. R., Rigaut, F., Roy, J. R., \& Draine, B. T. 2004, ApJ, 602, 770

Genzel, R., Eisenhauer, F., \& Gillessen, S. 2010, Reviews of Modern Physics, 82, 3121

Giannios, D. \& Sironi, L. 2013, MNRAS, 433, L25

Gillessen, S., Genzel, R., Fritz, T. K., et al. 2012, Nature, 481, 51

Gillessen, S., Plewa, P. M., Widmann, F., et al. 2019, ApJ, 871, 126

Ginsburg, I., Wang, X., Loeb, A., \& Cohen, O. 2016, MNRAS, 455, L21

Gravity Collaboration. 2018, A\&A, 618, L10

Low, F. J., Kleinmann, D. E., Forbes, F. F., \& Aumann, H. H. 1969, ApJL, 157, L97

Marrone, D. P., Moran, J. M., Zhao, J.-H., \& Rao, R. 2007, ApJL, 654, L57

Martins, F., Gillessen, S., Eisenhauer, F., et al. 2008, ApJL, 672, L119

Merritt, D. 2013, Dynamics and Evolution of Galactic Nuclei (Princeton: Princeton University Press)

Mužić, K., Eckart, A., Schödel, R., et al. 2010, A\&A, 521, A13

Mužić, K., Eckart, A., Schödel, R., Meyer, L., \& Zensus, A. 2007, A\&A, 469, 993

Narayan, R., Özel, F., \& Sironi, L. 2012, ApJL, 757, L20

Nayakshin, S. 2005, A\&A, 429, L33

Rauch, C., Mužić, K., Eckart, A., et al. 2013, A\&A, 551, A35

Rigaut, F., Geballe, T. R., Roy, J.-R., \& Draine, B. T. 2003, Astronomische Nachrichten Supplement, 324, 551

Roche, P. F., Lopez-Rodriguez, E., Telesco, C. M., Schödel, R., \& Packham, C. 2018, MNRAS, 476, 235

Różańska, A., Mróz, P., Mościbrodzka, M., Sobolewska, M., \& Adhikari, T. P. 2015, A\&A, 581, A64

Sabha, N., Zamaninasab, M., Eckart, A., \& Moser, L. 2014, in IAU Symposium, Vol. 303, The Galactic Center: Feeding and Feedback in a Normal Galactic Nucleus, ed. L. O. Sjouwerman, C. C. Lang, \& J. Ott, 150-152

Sanchez-Bermudez, J., Schödel, R., Alberdi, A., et al. 2014, A\&A, 567, A21

Schartmann, M., Burkert, A., \& Ballone, A. 2018, A\&A, 616, L8

Schödel, R., Feldmeier, A., Neumayer, N., Meyer, L., \& Yelda, S. 2014, Classical and Quantum Gravity, 31, 244007 
Shahzamanian, B., Eckart, A., Zajaček, M., et al. 2016, A\&A, 593, A131

Tanner, A., Ghez, A. M., Morris, M., et al. 2002, ApJ, 575, 860

Tanner, A., Ghez, A. M., Morris, M. R., \& Christou, J. C. 2005, ApJ, 624, 742

Viehmann, T., Eckart, A., Schödel, R., Pott, J. U., \& Moultaka, J. 2006, ApJ, 642, 861

Wang, Q. D., Nowak, M. A., Markoff, S. B., et al. 2013, Science, 341, 981

Wilkin, F. P. 1996, ApJL, 459, L31

Xu, Y.-D., Narayan, R., Quataert, E., Yuan, F., \& Baganoff, F. K. 2006, ApJ, 640, 319

Yuan, F., Quataert, E., \& Narayan, R. 2003, ApJ, 598, 301

Yusef-Zadeh, F. \& Morris, M. 1987, ApJ, 320, 545

Yusef-Zadeh, F. \& Morris, M. 1991, ApJL, 371, L59

Zajaček, M., Britzen, S., Eckart, A., et al. 2017a, A\&A, 602, A121

Zajaček, M., Eckart, A., Karas, V., et al. 2016, MNRAS, 455, 1257

Zajaček, M., Karas, V., Hosseini, E., et al. 2017b, in Proceedings of RAGtime 17-19: Workshops on black holes and neutron stars, 17-19/23-26 Oct., 1-5 Nov. 2015/2016/2017, Opava, Czech Republic, Z. Stuchlík, G. Török and V. Karas editors, Silesian University in Opava, 2017, ISBN 978-80-7510-257-7, ISSN 2336-5676, p. 237-252, 237-252

Zhang, Q. \& Zheng, X. 1997, ApJ, 474, 719

Zhao, J.-H., Morris, M. R., Goss, W. M., \& An, T. 2009, ApJ, 699, 186 